\title{
Casein Hydrolysate Containing Milk-Derived Peptides Improves Facial Pigmentation Independent of An Anti-Atherosclerosis Effect: A Randomized, Double-Blind, Placebo-Controlled Trial
}

\author{
Short running title: Casein hydrolysate improves facial pigmentation
}

\author{
Michiya Igase ${ }^{1,2}$,* , Yoko Okada ${ }^{1,2}$, Keiji Igase ${ }^{3}$, Masayuki Ochi ${ }^{2}$, Sayaka Matsumoto ${ }^{2}$, Kensuke \\ Senzaki ${ }^{2}$ and Yasumasa Ohyagi ${ }^{2}$ \\ 1 Department of Antiaging Medicine, Ehime University Graduate School of Medicine, Toon, Ehime, Japan \\ 2 Department of Geriatric Medicine and Neurology, Ehime University Graduate School of Medicine \\ 3 Department of Advanced Brain Therapy, Ehime University Graduate School of Medicine \\ * Correspondence to: migase@m.ehime-u.ac.jp
}

\begin{abstract}
Casein hydrolysate improves arterial stiffness, as estimated by brachial ankle pulse wave velocity (baPWV), in untreated hypertensive subjects. Facial pigmentation is a useful biomarker for arterial stiffness. This trial evaluated whether casein hydrolysate improves facial pigmentation in association with changes in arterial stiffness. A randomized, double-blind, placebo-controlled trial was conducted in 80 non-hypertensive Japanese participants randomly assigned to receive either active tablets containing casein hydrolysate or placebo for 48 weeks. Facial pigmentation and baPWV were measured at baseline and at the end of the intervention. Other biochemical atherosclerosis-related parameters were also measured, including advanced glycation end products (AGEs). Changes in facial pigmentation showed a significant difference between the groups. Change in baPWV was significantly better in the active than in the placebo group. In contrast, no significant association was seen between changes in facial pigmentation and those in baPWV. Among other atherosclerosis-related factors, changes in advanced glycation products (AGEs) were significantly decreased in the active compared to the placebo group. Further, changes in facial pigmentation were positively correlated with those in AGEs. Changes in AGEs were independently associated with changes in facial pigmentation. Casein hydrolysate improves facial pigmentation in non-hypertensive participants. Casein hydrolysate may have beneficial effects on glycation stress.
\end{abstract}

Keywords: casein hydrolysate ; Val-Pro-Pro ; Ile-Pro-Pro ; brachial ankle pulse wave velocity ; advanced glycation end products; facial pigmentation; A randomized; double-blind; placebo-controlled trial

\section{Introduction}

Although aging comes to everyone, the perceived age of the individual based on the appearance of the face is not the same as chronological age, and is in fact a better predictor of mortality rate than chronological age (1-3). In clinical findings, an association between perceived age and atherosclerosis has been identified, and might underlie previous findings that subjects who were evaluated as younger than their chronological age had significantly lower carotid arterial wall thickness. (4). 
Atherosclerosis involves two mechanisms, affecting arterial stiffness and vascular structure. Arterial stiffness is assessed by pulse wave velocity (PWV) and vascular structure by the intimamedia thickness (IMT) of the carotid artery. We previously used PWV and IMT to demonstrate that not only perceived age but also skin parameters, including the presence of facial pigmentation, might be useful biomarkers of atherosclerosis (5).

In this regard, advanced glycation end-products (AGEs) contribute to the development of atherosclerosis. Levels of accumulated AGE in the skin recently became measurable as skin autofluorescence (SAF) using a non-invasive apparatus, the autofluorescence reader. Significant associations have been identified between SAF and IMT as well as PWV (6).

A newly recognized casein hydrolysate which contains the milk-derived peptides Val-Pro-Pro (VPP) and Ile-Pro-Pro (IPP) has shown in vitro inhibitory activity against angiotensin converting enzyme (ACE). In several clinical trials conducted mainly in hypertensive and pre-hypertensive subjects, foods containing VPP and IPP improved PWV, which was in turn followed by a decrease in blood pressure (7). This result is widely known to derive not only from a simple hypotensive effect, but also from an effect on NO or similar (8). Given these results, casein hydrolysate containing VPP and IPP may improve perceived age in association with improvement in PWV or IMT in normotensive subjects.

Here, to determine whether casein hydrolysate improves facial pigmentation in association with changes in arterial stiffness, we investigated the association between these variables in a cohort of non-hypertensive Japanese adults.

\section{Materials and Methods}

\subsection{Study design}

A total of 80 healthy volunteers aged between 45 and 87 years (mean, $71.7 \pm 9.2$ years) who underwent a complete medical skin checkup at Ehime University's Anti-Aging Center between May 2017 and March 2019 were included. All participants underwent a medical examination, including fasting blood biochemistry, brachial blood pressure, central blood pressure, baPWV, IMT, SAF, and facial skin pigmentation at baseline and at the end of the 48-week intervention. The study was approved by institutional review board for clinical trial services at Ehime University Graduate School of Medicine (1704011). All participants provided written informed consent.

\subsection{Blood pressure}

Participants were seated and rested for 5 minutes in a quiet room, after which time blood pressure was measured over the brachial artery 3 times at 5-minute intervals using an HEM-9000AI (Omron Healthcare, Kyoto, Japan). The average of the last 2 measurements was recorded as representative of brachial blood pressure, in accordance with the ASCOT-CAFE study (9). Central blood pressure was also measured by HEM-9000AI.

\section{3. $P W V$}

Brachial-ankle pulse wave velocity (baPWV) was measured with a volume-plethysmographic apparatus (PWV/ABI; Omron Healthcare, Kyoto, Japan) and calculated as the time interval between the wave fronts of the brachial and ankle wave forms $(\Delta \mathrm{Tba})$ and the path length from the brachium to the ankle. Path lengths from the suprasternal notch to the brachium (Lb) and ankle (La) were obtained using the formulae $\mathrm{Lb}=0.2195 \times$ height +2.0734 and $\mathrm{La}=0.8129 \times$ height +12.328 . baPWV was then obtained as $(\mathrm{La}-\mathrm{Lb}) / \Delta \mathrm{Tba}(5)$.

\subsection{IMT}

To assess IMT, carotid arteries were examined by ultrasound using the 7.5-MHz probe of a Pro Sound $\alpha 10$ (Hitachi Aloka, Tokyo, Japan). Among the three layers on the B mode echograph of the 
far wall of the common carotid artery, the thickness of the two internal layers, which contain the hyper- and lower echoic layers, was designated as the IMT. IMT was measured at three contiguous sites at $1-\mathrm{cm}$ intervals proximal to the bulb from three directions each (anterior, lateral, and posterior) for the right and left sides. Results were averaged to obtain the mean IMTs for the right and left sides, which were in turn averaged again to obtain the mean overall IMT, which was used for analysis (10). Reproducibility (CV) of IMT was $3.3 \% \pm 1.6 \%$ for intra-measurement and $5.8 \pm 3.3 \%$ between measurements in our laboratory (11).

\subsection{Facial skin pigmentation}

Facial skin pigmentation was analysed using a Robo Skin Analyzer RSA50S II (MM\&NIIC, Tokyo, Japan). This system consists of a facial imaging capture enclosure picturizing box with a scattered light source and a high-fidelity digital camera. Three digital images of each participants' face (from the front, right 60 degrees oblique projection, and left 60 degrees oblique projection) were analyzed using computer software for the area marked by pigmentation. Facial skin pigmentation was evaluated as the total area of pigmented areas $>1.2 \mathrm{~mm}^{2}(5)$.

\section{6. $S A F$}

Accumulated levels of AGEs in the skin were estimated using the AGE-Reader (Diagno Optics Technologies BV, Groningen, the Netherlands), a desktop device that estimates skin levels using the characteristic fluorescent properties of certain AGEs. All measurements were performed at the volar side of the forearm of participants between 10 and $15 \mathrm{~cm}$ below the elbow, as described previously (12). Measurements were calculated by dividing the mean value of emitted light intensity per $\mathrm{nm}$ between 420 and $600 \mathrm{~nm}$ by the mean value of excitation light intensity per nm between 300 and 420 $\mathrm{nm}$, expressed in arbitrary units (AU). An earlier validation study showed an intra-individual Altman error percentage of $5.0 \%$ per day and $5.9 \%$ due to seasonal change (12).

\subsection{Test products}

The study was conducted under a randomized, double-blind, placebo-controlled design. The active products were tablets made from casein hydrolysate containing VPP and IPP, while the placebo products were prepared with sodium caseinate instead of casein hydrolysate. The amount of VPP and IPP in the active products was measured by liquid chromatography-mass spectrometry by Asahi Holdings Inc. (Tokyo, Japan). The active products were administered by single daily administration of 3 tablets, each containing $1.5 \mathrm{mg}$ of VPP and $1.9 \mathrm{mg}$ of IPP, taken in the morning after breakfast for 48 weeks (Table 1).

Table 1. Nutrient dose of active and placebo products.

\begin{tabular}{ccc}
\hline Component & Active & Placebo \\
\hline Calories (kcal/day) & 3.78 & 3.84 \\
\hline Moisture (\%) & 3.6 & 3.5 \\
\hline Protein (g/day) & 0.34 & 0.37 \\
\hline Fat (g/day) & 0.01 & 0.02 \\
\hline Carbohydrate (g/day) & 0.58 & 0.54 \\
\hline Sodium (mg/day) & 6 & 5 \\
\hline VPP (mg/day) & 1.5 & $\mathrm{ND}$ \\
\hline IPP (mg/day) & 1.9 & $\mathrm{ND}$
\end{tabular}

ND, not detected.

\subsection{Randomization}

Randomization was performed by an external statistician (CPCC, Tokyo, Japan) using the dynamic allocation procedure with a computer-generated list. Randomization was initiated in May 
2017 and completed in March 2018. Participants were instructed not to change their daily lifestyle, such as diet, physical activity etc. throughout the test period.

\section{Results}

\subsection{Demographic data and compliance}

A total of 80 participants were randomly assigned to the active product group (36 subjects) or placebo product group (44 participants). All randomized participants completed the 48 -week intervention, without dropouts. Mean compliance with test products among all participants was $99.0 \%$ (99.2\% in the active group and $98.8 \%$ in the placebo group). Table 2 shows the baseline characteristics of the randomized participants. Participants were well matched between the groups in terms of sex, age, body mass index (BMI) and blood biochemistry.

Table 2. Participant demographics at baseline.

\begin{tabular}{cccc}
\hline & All & Active & Placebo \\
\hline Number (male/female) & $80(33 / 47)$ & $36(13 / 23)$ & $44(20 / 24)$ \\
\hline Duration (weeks) & $47.3 \pm 5.3$ & $48.2 \pm 4.3$ & $46.5 \pm 5.9$ \\
\hline Age (years) & $71.7 \pm 9.2$ & $70.5 \pm 10.6$ & $72.6 \pm 7.8$ \\
\hline BMI $(\mathrm{kg} / \mathrm{m} 2)$ & $23.3 \pm 3.1$ & $22.1 \pm 2.2$ & $22.6 \pm 2.7$ \\
\hline sCr, mg/dL & $0.76 \pm 0.18$ & $0.76 \pm 0.18$ & $0.77 \pm 0.17$ \\
\hline BUN, mg/dL & $15.9 \pm 4.0$ & $15.3 \pm 4.1$ & $16.3 \pm 4.3$ \\
\hline eGFR, mL/min/1.73 m ${ }^{2}$ & $64.3 \pm 12.9$ & $67.4 \pm 14.8$ & $67.4 \pm 11.1$ \\
\hline LDL, mg/dL & $116.6 \pm 34.9$ & $116.8 \pm 32.3$ & $112.7 \pm 30.5$ \\
\hline HDL, mg/dL & $59.1 \pm 14.7$ & $60.5 \pm 13.0$ & $58.1 \pm 15.5$ \\
\hline TG, mg/dL & $97.5 \pm 39.4$ & $102.8 \pm 47.1$ & $105.6 \pm 62.1$ \\
\hline GOT, mg/dL & $24.0 \pm 5.6$ & $25.1 \pm 8.5$ & $30.1 \pm 5.6$ \\
\hline GPT, mg/dL & $19.4 \pm 7.4$ & $22.3 \pm 10.5$ & $19.1 \pm 7.6$ \\
\hline$\gamma$-GTP, cm/s & $32.2 \pm 24.4$ & $35.4 \pm 36.2$ & $31.2 \pm 22.1$ \\
\hline Hemoglobin, g/dL & $13.9 \pm 1.1$ & $14.1 \pm 1.2$ & $13.6 \pm 1.7$ \\
\hline Hematocrit, $\%$ & $41.0 \pm 3.2$ & $41.1 \pm 2.5$ & $40.7 \pm 3.6$ \\
\hline
\end{tabular}

Values are expressed as the mean \pm standard deviation. BMI, body mass index; sCr, serum creatinine; eGFR, creatinine-based estimated glomerular filtration rate; LDL, low-density lipoprotein; HDL, high-density lipoprotein; TG, serum triglyceride; GOT, glutamic oxaloacetic transaminase; GPT, glutamic pyruvate transaminase; $\gamma$-GTP, $\gamma$-glutamyltransferase.

\subsection{Changes in blood biochemistry and adverse events}

No significant differences between the groups were seen in changes in blood biochemistry variables (Table 2).

\subsection{Changes in hemodynamic variables and atherosclerosis markers}

No significant differences between the groups were seen in other hemodynamic parameters at baseline (Table 3). Changes in hemodynamic parameters after the 48-week intervention are also shown in Table 3. In this trial, reductions in brachial SBP from baseline were seen in both the active products group $(-0.1 \% \pm 11.1 \%)$ and placebo products group $(1.1 \% \pm 11.0 \%)$, albeit without significant differences between them. Changes in central SBP measured by HEM-9000AI also showed no significant difference between the groups (Table 3). Percent change in baPWV was significantly greater in the active products group than in the placebo products group $(-0.47 \% \pm 8.41 \%$ vs $5.09 \% \pm 12.31 \%, \mathrm{P}=0.024)$. However, no significant association was seen between changes in mean overall IMT (Table 3).

Table 3. Change in BP, PR and baPWV during treatment. 


\begin{tabular}{|c|c|c|c|}
\hline & Baseline & 48 Weeks & Change (\%), week 48 \\
\hline \multicolumn{4}{|c|}{ Brachial SBP, mmHg } \\
\hline $\operatorname{LTP}(\mathrm{n}=36)$ & $129 \pm 17$ & $128 \pm 14$ & $-0.1 \pm 11.1$ \\
\hline Placebo $(n=44)$ & $127 \pm 17$ & $127 \pm 16$ & $1.1 \pm 11.0$ \\
\hline \multicolumn{4}{|c|}{ Brachial DBP, $\mathrm{mmHg}$} \\
\hline $\operatorname{LTP}(\mathrm{n}=36)$ & $73 \pm 13$ & $75 \pm 11$ & $3.5 \pm 11.9$ \\
\hline Placebo $(\mathrm{n}=44)$ & $70 \pm 9$ & $73 \pm 9$ & $4.7 \pm 12.4$ \\
\hline \multicolumn{4}{|l|}{$\mathrm{PR}, \mathrm{bpm}$} \\
\hline $\operatorname{LTP}(n=36)$ & $65 \pm 8$ & $65 \pm 11$ & $0.3 \pm 11.8$ \\
\hline Placebo $(n=44)$ & $64 \pm 9$ & $65 \pm 12$ & $1.3 \pm 7.8$ \\
\hline \multicolumn{4}{|l|}{$\mathrm{CSBP}, \mathrm{mmHg}$} \\
\hline $\operatorname{LTP}(n=36)$ & $138 \pm 18$ & $136 \pm 15$ & $-0.5 \pm 11.9$ \\
\hline Placebo $(n=44)$ & $135 \pm 18$ & $136 \pm 18$ & $1.2 \pm 11.2$ \\
\hline \multicolumn{4}{|l|}{$\mathrm{baPWV}, \mathrm{cm} / \mathrm{s}$} \\
\hline $\operatorname{LTP}(n=36)$ & $1596 \pm 297$ & $1575 \pm 238$ & $-0.47 \pm 8.41^{*}$ \\
\hline Placebo $(\mathrm{n}=44)$ & $1574 \pm 386$ & $1636 \pm 346$ & $5.09 \pm 12.31$ \\
\hline \multicolumn{4}{|l|}{ Carotid IMT, mm } \\
\hline $\operatorname{LTP}(n=36)$ & $0.80 \pm 0.16$ & $0.77 \pm 0.12$ & $-2.77 \pm 8.94$ \\
\hline Placebo $(n=44)$ & $0.81 \pm 0.12$ & $0.76 \pm 0.09$ & $-5.42 \pm 9.31$ \\
\hline
\end{tabular}

\subsection{Changes in facial pigmentation}

No significant difference in facial pigmentation area was seen at baseline between the active product and placebo groups. After 48 weeks of treatment, however, a significant difference in changes in facial pigmentation between the groups was observed (active $-1.41 \% \pm 1.17 \%$ [ $n=36]$ vs placebo $-0.66 \% \pm 1.44 \%[\mathrm{n}=44] ; \mathrm{P}=0.014$ ) (Figure 1 ).

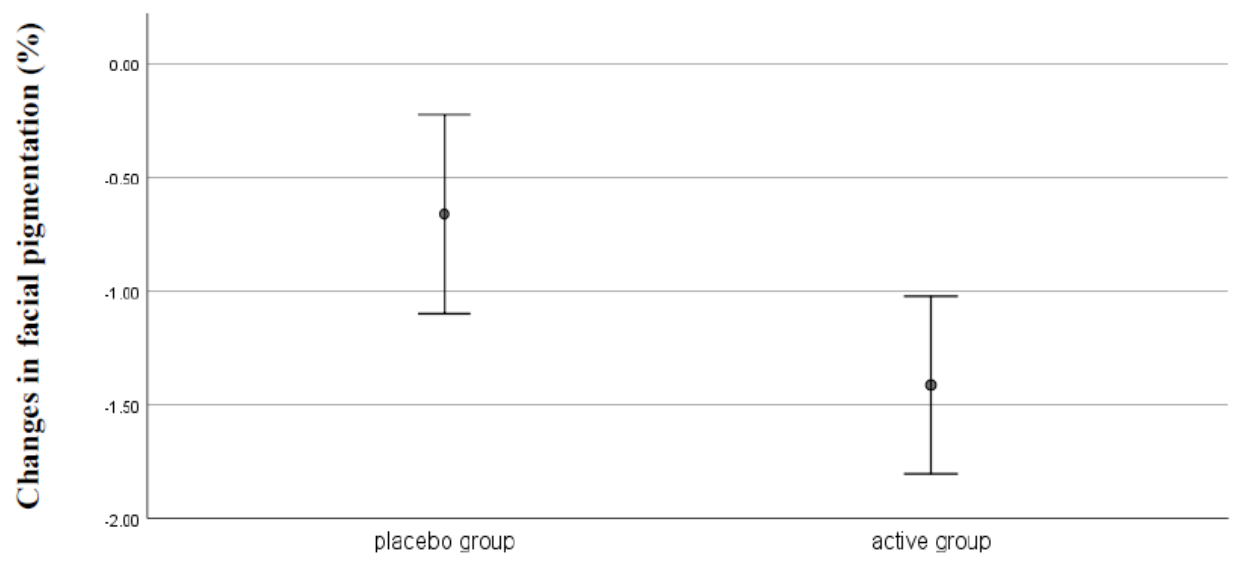

Figure 1. Comparison of percent changes in facial pigmentation between groups.

\subsection{Relationship between changes in facial pigmentation and PWV}

No significant association was seen between changes in facial pigmentation and PWV (Figure 2). 


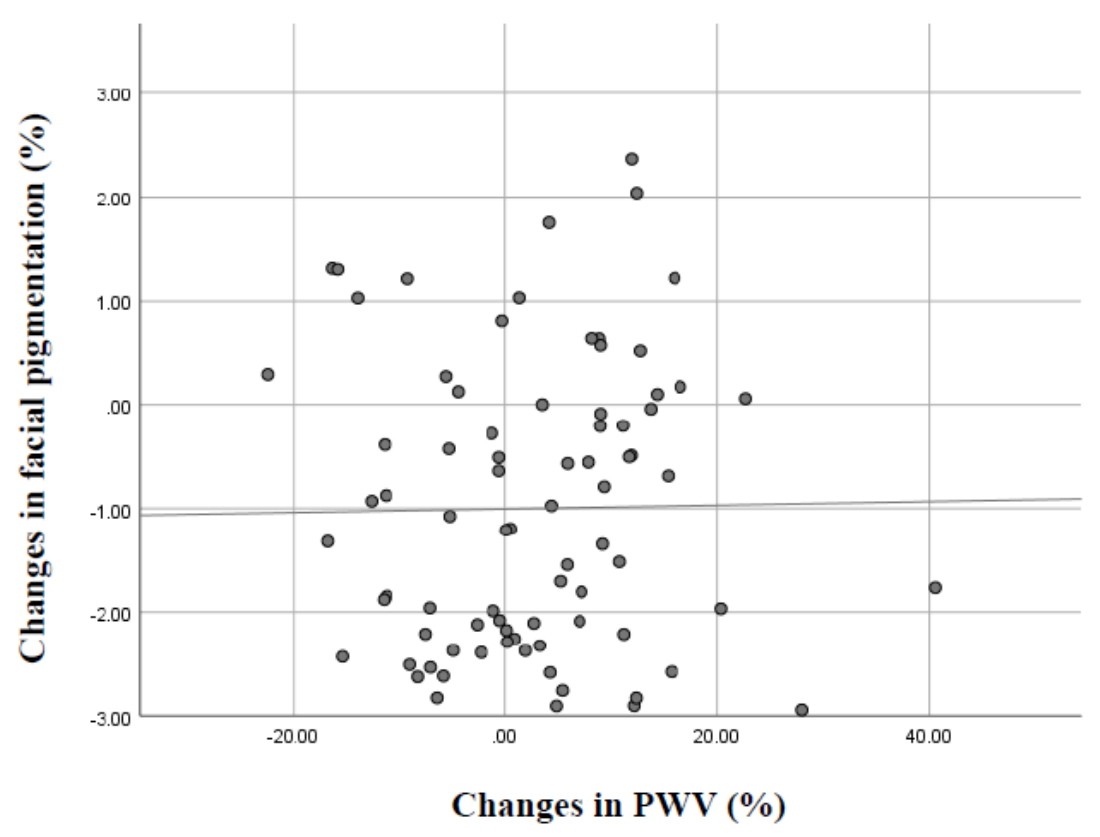

Figure 2. Relationship between changes in facial pigmentation and changes in PWV.

\subsection{Changes in facial pigmentation and SAF}

A positive correlation was seen between changes in facial pigmentation and advanced glycation products (AGEs) (Figure 3).

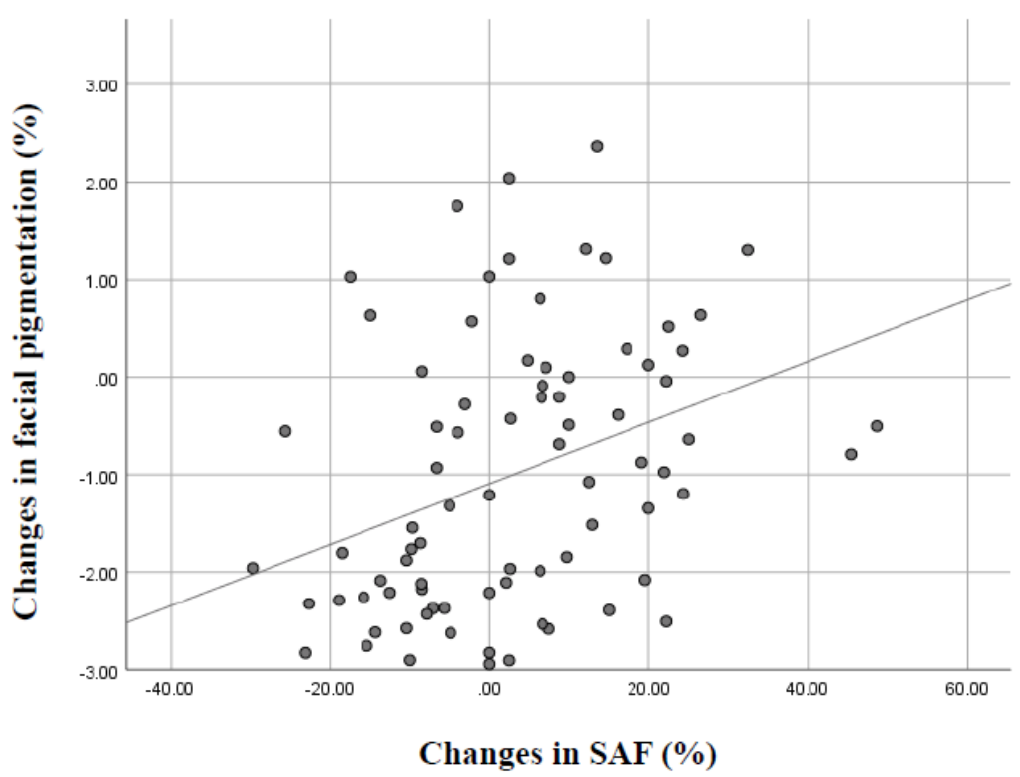

Figure 3. Relationship between changes in facial pigmentation and changes in SAF.

\subsection{Multiple regression analysis for changes in facial pigmentation}

The association of AGEs and other parameters with facial pigmentation was analysed multiple regression analyses. Changes in AGEs were independently associated with changes in facial pigmentation independently of age and changes in Hba1c (Table 4).

Table 4. Multiple regression analysis for change in facial pigmentation. 


\begin{tabular}{ccc}
\hline Factor & $\boldsymbol{\beta}$ & $\mathbf{p}$ \\
\hline Age, years & -0.079 & 0.468 \\
\hline Changes in Hba1c, \% & 0.038 & 0.727 \\
\hline Changes in AGEs, \% & 0.354 & 0.002 \\
\hline
\end{tabular}

AGEs, advanced glycation endproducts4. Discussion.

\subsection{Effect of VPP and IPP on arterial stiffness in normotensive subjects}

In their recent 8-week intervention study in pre-hypertensive and hypertensive subjects, Nakamura et al demonstrated that VPP and IPP reduced blood pressure, followed by an improvement in arterial stiffness. These results indicated that VPP and IPP have an anti-atherosclerotic effect beyond a hypotensive effect. The authors speculated that this effect was partly influenced by NO synthesis via ACE inhibition (13). In our present study also, although baPWV decreased significantly in the active products group, change in brachial SBP from baseline among normotensive subjects was almost identical between the groups. The improvement in baPWV after long-term intervention in our trial suggests that VPP and IPP may have induced a functional change rather than a vascular structural change as estimated by carotid IMT. The atherosclerotic risk factors associated with each of the two parameters can therefore be expected to differ. To our knowledge, this is the first study to demonstrate differences in the associations between IMT and baPWV with the effect of lactotripeptide in the same participants.

\subsection{Effect of VPP and IPP on facial pigmentation}

In this study, we sought to demonstrated that the area of facial pigmentation was independently associated with baPWV in middle-aged to elderly non-hypertensive Japanese subjects. Although there was no significant difference in facial pigmentation area between the active products and placebo groups at baseline, facial pigmentation area after 48-week treatment was significantly reduced in the active products group compared to the placebo products group. Nevertheless, we saw no correlation between changes in baPWV and facial pigmentation, and therefore no close relationship between facial pigmentation - commonly used to estimate perceived age - and atherosclerosis in this study.

\subsection{Changes in facial pigmentation and AGEs}

Although we were unable to explain the lack of association between these two parameters, we did newly identify a correlation between facial pigmentation and AGEs as estimated by SAF. Accumulation of AGEs accelerates the multisystem functional decline that occurs with aging, and therefore contributes to the aging phenotype (14). Accumulation of AGEs is linked with many degenerative processes or disorders, including aging and atherosclerosis. In addition, AGE production in skin cells is known to promote stiffness and loss of elasticity through their buildup in connective tissue (15). Thus, AGE-mediated melanogenesis may hold promise as a novel mechanism of age-related skin pigmentation.

\subsection{Limitations}

Several limitations of the study warrant mention. First, the sample size of this study was small, and confirmation of our findings in a large cohort study is needed. Second, our program participants were fitness-conscious, and no heavy smokers or heavy alcohol drinkers were included. The milk-derived peptides VPP and IPP might have a greater effect in preventing AGE-related pigmentation when combined with lifestyle interventions.

\section{Conclusions}

We confirmed for the first time that a 48-week intervention with casein hydrolysate containing the milk-derived peptides VPP and IPP significantly improved facial pigmentation area in 
association with AGE changes as compared with placebo. In addition to their well-known beneficial effects on arterial properties, repeated intake of foods containing VPP and IPP might also have beneficial esthetic effects.

Author Contributions: Conceptualization, M.I.; Methodology, S.M.; Software, M.I.; Validation, M.I.; Formal Analysis, K.I.; Investigation, M.O.; Resources, M.I.; Data Curation, Y.O.; Writing - Original Draft Preparation, M.I.; Writing - Review \& Editing, Y.O.; Visualization, M.I.; Supervision, Y.O.; Project Administration, K.S.; Funding Acquisition, M.I.

Acknowledgments: This study was supported by a Grant-in-Aid for Scientific Research from the Ministry of Education, Culture, Sports, Science and Technology of Japan (18K10105).

Conflicts of Interest: All authors have no conflicts of Interest.

\section{References}

1. Nkengne A, Bertin C, Stamatas GN,Giron A, Rossi A, Issachar N, Fertil B. Influence of facial skin attributes on the perceived age of Caucasian Women. J Eur Acad Dermatol Venereol. 2008; 22: 982-991.

2. Mayes AE, Murray PG, Gunn DA, Tomlin CC, Catt SD, Wen YB, Zhou LP, Wang HQ, Catt M, Granger SP. Ageing appearance in China: biophysical profile of facial

skin and its relationship to perceived age. J Eur Acad Dermatol Venereol. 2010; 24: 341-348.

3. Christensen K, Thinggaard M, McGue M, Rexbye H, Hjelmborg JV, Aviv A, Gunn D, van der Ouderaa F, Vaupel JW. Perceived age as clinically useful biomarker of ageing: cohort study. BMJ. 2009; 339: b5262.

4. Kido M, Kohara K, Miyawaki S, Tabara Y, Igase M, Miki T. Perceived age of facial features is a significant diagnosis criterion for age-related carotid atherosclerosis in Japanese subjects: J-SHIPP study. Geriatr Gerontol Int. 2012; 12: 733-40.

5.Miyawaki S, Kohara K, Kido T, Tabara Y, Igase M, Miki T, Sayama K. Facial pigmentation as a biomarker of carotid atherosclerosis in middle-aged to elderly healthy Japanese subjects. Skin Res Technol. 2016; 22: 20-24.

6. Ninomiya H, Katakami N, Sato I, Osawa S, Yamamoto Y, Takahara M, Kawamori D, Matsuoka TA, Shimomura I. Association between Subclinical Atherosclerosis Markers and the Level of Accumulated Advanced Glycation End-Products in the Skin of Patients with Diabetes. J Atheroscler Thromb. 2018; 25: 1274-1284.

7. Cicero AF, Gerocarni B, Laghi L, Borghi C. Blood pressure lowering effect of lactotripeptides assumed as functional foods: a meta-analysis of current available clinical trials. J Hum Hypertens. 2011; 25: 425-36.)

8. Hirota T, Ohki K, Kawagishi R, Kajimoto Y, Mizuno S, Nakamura Y, Kitakaze M. Casein hydrolysate containing the antihypertensive tripeptides Val-Pro-Pro and Ile-Pro-Pro improves vascular endothelial function independent of blood pressure-lowering effects: contribution of the inhibitory action of angiotensin-converting enzyme. Hypertens Res. 2007; 30: 489-496.

9. Williams B, Lacy PS, Thom SM, et al. Differential impact of blood pressure-lowering drugs on central aortic pressure and clinical outcomes: principal results of the Conduit Artery Function Evaluation (CAFE) study. Circulation. 2006; 113: 1213-1225

10.Ochi N, Kohara K, Tabara Y et al. Association of central systolic blood pressure with intracerebral small vessel disease in Japanese. Am J Hypertens. 2010; 23: 889-894.).

11. Kido M, Kohara K, Miyawaki S, Tabara Y, Igase M, Miki T. Perceived age of facial features is a significant diagnosis criterion for age-related carotid atherosclerosis in Japanese subjects: J-SHIPP study. Geriatr Gerontol Int. 2012; 12: 733-740.)

12. Meerwaldt R, Graaff R, Oomen PH, Links TP, Jager JJ, Alderson NL, Thorpe SR, Baynes JW, Gans RO, Smit AJ. Simple non-invasive assessment of advanced glycation endproduct accumulation. Diabetologia. 2004; 47: 1324-1330.

13. Tanaka H, Munakata M, Kawano Y, et al. Comparison between carotid-femoral and brachial-ankle pulse wave velocity as measures of arterial stiffness. J Hypertens. 2009; 27:2022-7.

14. Semba RD, Nicklett EJ, Ferrucci L. Does accumulation of advanced glycation end products contribute to the aging phenotype? J Gerontol A Biol Sci Med Sci. 2010; 65: 963-75. 
9 of 9

15. Lee EJ, Kim JY, Oh SH. Advanced glycation end products (AGEs) promote melanogenesis through receptor for AGEs. Sci Rep. 2016; 6: 27848. doi: 10.1038/srep27848. 